\title{
THE KNAPP-STEIN DIMENSION THEOREM FOR $p$-ADIC GROUPS ${ }^{1}$
}

\author{
ALLAN J. SILBERGER
}

\begin{abstract}
Knapp and Stein have proved for semisimple Lie groups that the dimension of the commuting algebra of an induced tempered representation equals the index of a certain reflection group in a larger group. A precise analogue of their result is stated and proved in this paper for $p$-adic groups.
\end{abstract}

The purpose of this paper is to prove for $p$-adic groups the analogue of a theorem due to Knapp and Stein [3] in the case of real semisimple Lie groups. The Knapp-Stein theorem has precisely-mutatis mutandis-the same statement as we give below. Our proof, which depends upon the Harish-Chandra commuting algebra theorem [4, Theorem 5.5.3.2], carries over, with only slight changes, to the real case too.

We wish to thank Nolan Wallach for useful discussions.

1. Some terminology. Let $\Omega$ be a nonarchimedean local field and $\mathbf{G}$ a connected reductive $\Omega$-group. Let $G$ denote the group of $\Omega$-points of $\mathbf{G}$. In this paper we employ the terminology and notations of [2] and [4].

Fix a minimal $p$-pair $\left(P_{0}, A_{0}\right)\left(P_{0}=M_{0} N_{0}\right)$ of $G$ and an $A_{0}$-good maximal compact subgroup $K$ of $G$. Let $(P, A)(P=M N)$ be a semistandard $p$-pair of $G$. Let $a^{*}$ denote the real Lie algebra of $A$. Let $W$ denote the factor group $N_{G}(A) / M$. Assume that $\mathrm{a}^{*}$ has a $W$-invariant scalar product defined on it. Let $\Sigma_{r}=\Sigma_{r}(P, A)$ denote the set of positive reduced $A$-roots, $\Sigma^{0}(P, A)$ the subset consisting of the simple $A$-roots.

Let $\sigma \in \omega \in \mathcal{E}_{2}(M)$. Let $W(\omega)=\left\{s \in W \mid \omega^{s}=\omega\right\}$. Let $\mu(\omega: \nu)\left(\nu \in a^{*}\right)$ denote the Harish-Chandra function associated to $\omega$ and $G$ [2, Theorem 20], $[4, \S 5.4 .3]$. It is proved in [4, Corollary 5.4.3.3] (cf. [2, Theorem 24]) that, with $c>0$,

$$
c \mu(\omega: \nu)=\prod_{\alpha \in \Sigma_{r}} \mu_{\alpha}(\omega: \nu)
$$

where $\mu_{\alpha}(\omega: \nu)$ is the Harish-Chandra function associated to $\omega$ and $M_{\alpha}=$ $Z_{G}\left(A_{\alpha}\right)\left(A_{\alpha}\right.$ is the maximal subtorus of $A$ in the kernel of the root character $\left.\xi_{\alpha}\right)$. A root $\alpha \in \Sigma_{r}$ is called $\omega$-special if $\mu_{\alpha}(\omega: 0)=0$. If $\alpha$ is $\omega$-special, then

Received by the editors June 21, 1977.

AMS (MOS) subject classifications (1970). Primary 22E50.

Key words and phrases. Reductive $p$-adic groups, tempered unitary representations, commuting algebras.

${ }^{1}$ Research partially supported by NSF Grant MCS 76-11624 A01.

() American Mathematical Society 1978 
there is a reflection $s_{\alpha} \in W(\omega)$. Let $\Sigma^{\prime \prime}= \pm\left\{\alpha \in \Sigma_{r}: \alpha \omega\right.$-special $\}$. Then $\Sigma^{\prime \prime}$ is a root system in a subspace of $a^{*}\left[1, \mathrm{VI}, \S 2\right.$, Proposition 9]. We write $W^{\prime \prime}(\omega)$ for the Weyl group of this root system; $W^{\prime \prime}(\omega)$ is the subgroup of $W(\omega)$ generated by the set $\left\{s_{\alpha}: \alpha \omega\right.$-special $\}$.

Let $C_{M}^{G}(\omega)$ denote the class of the induced representation $\pi_{P, \omega}=$ $\operatorname{Ind}_{P}^{G}\left(\delta_{P}^{1 / 2} \sigma\right)$. Then $C_{M}^{G}(\omega)$ is unitary and independent of the choice of $P \in$ $\mathscr{P}(A)$ or $\omega$ in a $W$-orbit.

2. The theorem. In the following we assume that the $c$-functions and ${ }^{0} c$ functions, as well as the space $L(\omega, P)$, are associated to a fixed smooth unitary double representation of $K$ which satisfies associativity conditions.

THEOREM. The commuting algebra of the class $C_{M}^{G}(\omega)$ has dimension [ $W(\omega)$ : $\left.W^{\prime \prime}(\omega)\right]$

Proof. Harish-Chandra's commuting algebra theorem implies that, for any $P \in \mathcal{P}(A)$, the mapping $s \mapsto{ }^{0} c_{P \mid P}(s: \omega)$, a homomorphism from $W(\omega)$ to the group of unitary automorphisms of the algebra $L(\omega, P)$, may be regarded as a mapping onto a set of generators for the commuting algebra of $\operatorname{Ind}_{P}^{G}\left(\delta_{P}^{1 / 2} \sigma\right)(\sigma \in \omega)$. We prove the theorem in two steps: (1) ${ }^{0} c_{P \mid P}(s: \omega)$ is the identity on $L(\omega, P)$ when $s \in W^{\prime \prime}(\omega)$; (2) the dimension of the commuting algebra is at least $\left[W(\omega): W^{\prime \prime}(\omega)\right]$.

For (1) it is enough to show that ${ }^{0} c_{P \mid P}(s: \omega)=I$ whenever $s$ is the reflection $s_{\alpha}$ associated to an $\omega$-special root $\alpha$. Given any such $\alpha$, we may choose $P_{1} \in \mathcal{P}(A)$ such that $\alpha \in \Sigma^{0}\left(P_{1}, A\right)$. It is enough to show that

$$
{ }^{0} c_{P_{1} \mid P_{1}}(\bar{s}: \omega)=I \text {, }
$$

since

$$
\begin{aligned}
{ }^{0} c_{P \mid P}(s: \omega) & ={ }^{0} c_{P \mid P_{1}}(1: \omega){ }^{0} c_{P_{1} \mid P_{1}}(s: \omega){ }^{0} c_{P_{1} \mid P}(1: \omega) \text { and } \\
I & ={ }^{0} c_{P \mid P}(1: \omega)={ }^{0} c_{P \mid P_{1}}(1: \omega){ }^{0} c_{P_{1} \mid P}(1: \omega) ;
\end{aligned}
$$

both relations follow from the general transformation formulas for the ${ }^{0} c$ functions [2, §§11-12], [4, §5.2.4].

Thus, without loss of generality, assume that $\alpha \in \Sigma^{0}(P, A)$. Let $A_{\alpha}$ and $M_{\alpha}$ be as before. Then $P \cap M_{\alpha}={ }^{*} P_{\alpha}$ is a maximal parabolic subgroup of $M_{\alpha}$. Since $\mu_{\alpha}(\omega: 0)=0$, the representation $\operatorname{Ind}_{* P_{\alpha}}^{M_{\alpha}}\left(\delta *_{P_{\alpha}}^{1 / 2} \sigma\right)$ is irreducible, so ${ }^{0} c * P_{\alpha} \mid{ }^{*} P_{\alpha}\left(s_{\alpha}: \omega\right)=I_{L\left(\omega, * P_{\alpha}\right)}$. On the other hand, by [4, Theorem 5.3.5.3(4)],

$$
{ }^{0} c_{P \mid P}\left(s_{\alpha}: \omega\right)={ }^{0} c * P_{\alpha}\left|* P_{\alpha}\left(s_{\alpha}: \omega\right)\right|_{L(\omega, P)},
$$

so ${ }^{0} c_{P \mid P}(s: \omega)=I_{L(\omega, P)}$ for all $s \in W^{\prime \prime}(\omega)$, as required.

To prove (2) we shall argue as follows. Let $\pi_{P, \omega}=\operatorname{Ind}_{P}^{G}\left(\delta_{P}^{1 / 2} \sigma\right)$ act in a vector space $\mathcal{H}$. Consider the tempered Jacquet module $\overline{\mathcal{H}}={ }_{w}(\mathcal{H} / \mathcal{H}(\bar{P}))$ associated to $\pi_{P, \omega}$, with $\bar{\pi}_{P, \omega}$ the representation of $M$ on $\overline{\mathcal{H}}$. It is known [4, Theorem 5.4.1.1] that $\overline{\mathcal{K}}$ has a composition series of length $[W(G / A)]$, whose composition factors, counted with multiplicities, are $\left\{\delta_{P}^{1 / 2} \omega^{s}\right\}_{s \in W(G / A)}$. Furthermore, it follows from the fact that discrete series are projectives in the 
category of tempered modules (with a fixed central exponent) that $\overline{\mathcal{H}}$ is a direct sum of isotypic submodules. Let $\overline{\mathcal{H}}(\omega)$ be the submodule all of whose components are of class $\delta_{\bar{P}}^{1 / 2} \omega$. The composition series for $\overline{\mathcal{H}}(\omega)$ has length $[W(\omega)]$. The Frobenius reciprocity theorem [4, Theorem 1.7.10] implies that $\delta_{\bar{P}}^{1 / 2} \omega$ occurs as a quotient in $\overline{\mathcal{H}}(\omega)$ a number of times equal to the dimension of the commuting algebra of $C_{M}^{G}(\omega)$. Thus, to prove (2), it is sufficient to show that $\overline{\mathcal{F}}(\omega)$ contains $\delta_{\bar{P}}^{1 / 2} \omega$ as a quotient at least $\left[W(\omega): W^{\prime \prime}(\omega)\right]$ times. For this, it is obviously sufficient to show that the multiplicity of the central character $\delta_{\bar{P}}^{1 / 2} \chi_{\omega}$ in $\overline{\mathcal{F}}(\omega)$ is no greater than [ $\left.W^{\prime \prime}(\omega)\right]$.

We shall prove, instead, an equivalent fact involving the Eisenstein integral and the weak constant term. Let $\psi \in L(\omega, P)$ and consider the Eisenstein integral $E(P: \psi: \nu)$. The weak constant term ${ }_{w} E_{P}(P: \psi: \nu)$ is holomorphic in a neighborhood $U$ of $a^{*}[4$, Corollary 5.3.3.5]. For $\nu \in U$ in general position we may write

$$
{ }_{w} E_{P}(P: \psi: \nu)=\sum_{s \in W(G / A)} c_{P \mid P}(s: \omega: \nu) \psi \chi_{s \nu} .
$$

For any $s \in W(G / A)$, the function

$$
c_{P \mid P}(s: \omega: \nu)=s c_{P^{s^{-} \mid P}}(1: \omega: \nu)=s \prod_{\alpha \in \Sigma_{r}(P, A)} c_{\alpha}^{ \pm}(1: \omega: \nu),
$$

where each function $c_{\alpha}^{+}(1: \omega: \nu)$ or $c_{\alpha}^{-}(1: \omega: \nu)$ is a $c$-function associated to a pair $\left(M_{\alpha}, M\right)$ in which $M_{\alpha}$ is a reductive subgroup of $G$ containing $\left(P \cap M_{\alpha}, A\right)$ as a maximal $p$-pair $\left[4, \S 5.4\right.$.3]. Each function $c_{\alpha}^{ \pm}$is essentially a meromorphic function of a single complex variable, holomorphic for all $\nu \in U$, unless $\alpha$ is an $\omega$-special root; if $\alpha$ is an $\omega$-special root, then the hyperplane $H_{\alpha}$ passing through $\nu=0$ and orthogonal to $\alpha$ is singular for $c_{\alpha}^{ \pm}$. This implies that the function $c_{P \mid P}(s: \omega: \nu)$ is holomorphic on $U-$ $\bigcup_{\alpha \in \Sigma^{\prime \prime}} H_{\alpha}$.

We claim that, to prove (2), it is sufficient to show that the function

$$
\Phi\left(s_{0}, \nu\right)=\sum_{s \in W^{\prime \prime}(\omega)} c_{P \mid P}\left(s s_{0}: \omega: \nu\right) \psi \chi_{s s_{0} \nu}
$$

is holomorphic at $\nu=0$ for any $s_{0} \in W(\omega)$. If this is so, then one can show exactly as in [4, §§5.3.2-3] (and we shall not give the details here) that $\Pi_{t \in W^{\prime \prime}(\omega)}\left(\chi_{t s_{0} \nu}(a)-\rho(a)\right) \Phi\left(s_{0}, \nu\right)$ is identically zero near $\nu=0$ and, as a consequence, that the multiplicity of the exponent $\chi_{\omega}$ is no greater than $\left[W^{\prime \prime}(\omega)\right]$. However, by [4, Corollary 3.2.5(3)], the multiplicity of the exponent $\chi_{\omega}$ related to the constant term is the same as the multiplicity of $\delta_{\bar{P}}^{1 / 2} \chi_{\omega}$ in the Jacquet space. Thus, it follows easily that, since $\delta_{\vec{P}}^{1 / 2} \omega$ occurs $[W(\omega)]$ times in the composition series of $\overline{\mathcal{H}}(\omega), \delta_{\bar{P}}^{1 / 2} \omega$ actually occurs as a quotient at least [ $\left.W(\omega): W^{\prime \prime}(\omega)\right]$ times, as required.

Let us show that $\Phi\left(s_{0}, \nu\right)$ is holomorphic at $\nu=0$. It is enough to check this for any $\psi \in L(\omega, P)$. As is well known, we may (and do) choose $\psi$ such that $E(P: \psi: \nu)=E(P: \psi: s \nu)$ for all $s \in W(\omega)$ and $\nu \in a^{*}$. Observe that, in this 
case, $c_{P \mid P}(s: \omega: t \nu) \psi \chi_{s t \nu}=c_{P \mid P}(s t: \omega: \nu) \psi \chi_{s t \nu}$ for all $s, t \in W(\omega)$ and $\nu \in$ $a^{*}$, so $\Phi\left(1, s_{0} \nu\right)=\Phi\left(s_{0}, \nu\right)$. Thus, it is sufficient to check that $\Phi(1, \nu)$ is holomorphic at $\nu=0$.

We shall need the fact that the weak constant term takes its image in the direct sum $\bigoplus_{s \in W / W\left(\omega_{s}\right)} \mathbb{Q}\left(M, \tau_{M}\right)_{\omega_{s s}^{s}}$. This is proved in the supercuspidal case in [4, Corollary 5.4.4.6]; the proof in the present case is exactly the same and depends upon the fact, used above, that discrete series are projectives in the category of tempered admissible modules. As a consequence, any term

$$
E_{P, \omega_{\nu_{0}}}(P: \psi: \nu)=\sum_{s \in W\left(\omega_{\nu_{0}}\right)} E_{P, s}(P: \psi: \nu)
$$

is holomorphic in a neighborhood of $\nu=\nu_{0}$.

We have already observed that the singularities of $\Phi(1, \nu)$, if there are any, lie in $\cup H_{\alpha}\left(\alpha \in \Sigma^{\prime \prime}\right)$. It follows easily from the Weierstrass Preparation Theorem that a nonempty zero set of a holomorphic function defined in an open set $U$ of a complex space is a union of hypersurfaces in $U$. Therefore, it is sufficient, in order to show that $\Phi(1, \nu)$ is holomorphic at $\nu=0$, to show that the singularities lie in a subset of codimension at least two.

Let $\alpha \in \Sigma^{\prime \prime}$ and $\nu_{0} \in H_{\alpha}-\cup_{\alpha^{\prime} \neq \alpha} H_{\alpha^{\prime}}$. We shall show that $\Phi(1, \nu)$ is holomorphic at $\nu=\nu_{0}$. To see this, note first that $W\left(\omega_{\nu_{0}}\right) \cap W^{\prime \prime}\left(\omega_{0}\right)=$ $\left\{1, s_{\alpha}\right\}$, which follows from well-known properties of Weyl groups. We may choose representatives $s_{1}, \ldots, s_{r} \in W^{\prime \prime}(\omega) \backslash W(\omega)$ such that $s_{i}$ and $s_{\alpha} s_{i}$ fix $H_{\alpha}$ for all $i=1, \ldots, r$. There is a neighborhood $V$ of $\nu_{0}$ on which

$$
E_{P, \omega_{\nu_{0}}}(P: \psi: \nu)=\sum_{i=1}^{r}\left(c_{P \mid P}\left(s_{i}: \omega: \nu\right) \psi \chi_{s_{i} \nu}+c_{P \mid P}\left(s_{\alpha} s_{i}: \omega: \nu\right) \psi \chi_{s_{\alpha} s_{i} \nu}\right)
$$

is holomorphic. For all $\nu \in V \cap H_{\alpha}$ and $i=1, \ldots, r$. ·

$$
\begin{aligned}
c_{P \mid P}\left(s_{i}: \omega: \nu\right) & \psi \chi_{s_{i} \nu}+c_{P \mid P}\left(s_{\alpha} s_{i}: \omega: \nu\right) \psi \chi_{s_{\alpha} s_{i} \nu} \\
& =c_{P \mid P}(1: \omega: \nu) \psi \chi_{\nu}+c_{P \mid P}\left(s_{\alpha}: \omega: \nu\right) \psi \chi_{s_{\alpha} \nu},
\end{aligned}
$$

from which it follows that $c_{P \mid P}(1: \omega: \nu) \psi \chi_{\nu}+c_{P \mid P}\left(s_{\alpha}: \omega: \nu\right) \psi \chi_{s_{\alpha} \nu}$ and, hence, $\Phi(1, \nu)$ is holomorphic near $\nu=\nu_{0}$. We conclude that $\Phi(1, \nu)$ is, in fact, holomorphic at $\nu=0$. This proves the theorem.

\section{REFERENCES}

1. N. Bourbaki, Éléments de mathématique, Fasc. XXXIV. Groupes et algèbres de Lie, Chapters IV, V, VI, Actualités Sci. Indust., no. 1337, Hermann, Paris, 1968.

2. Harish-Chandra, Harmonic analysis on reductive p-adic groups, Proc. Sympos. Pure Math., vol. 26, Amer. Math. Soc., Providence, R. I., 1974, pp. 167-192.

3. A. W. Knapp and E. M. Stein, Singular integrals and the principal series. IV, Proc. Nat. Acad. Sci. U.S.A. 72 (1975), 2459-2461.

4. A. J. Silberger, Introduction to harmonic analysis on reductive p-adic groups, based on lectures by Harish-Chandra (to appear).

Department of Mathematics, Cleveland State University, Cleveland, Oho 44115 